\title{
Enhanced QoS and QoE Support through Energy Efficient Handover Algorithm for UMTS Architectures
}

\author{
Mr. A. Kingsly Jabakumar \\ Assistant professor-ECE, SCAD Institute of Technology, Palladam, India \\ kingslyjkumar@gmail.com
}

\begin{tabular}{|c|c|}
\hline Article History & Abstract \\
\hline $\begin{array}{l}\text { Article Submission } \\
19 \text { November } 2018 \\
\text { Revised Submission } \\
17 \text { January } 2019 \\
\text { Article Accepted } \\
1 \text { March } 2019 \\
\text { Article Published } \\
31^{\text {st }} \text { March } 2019\end{array}$ & $\begin{array}{l}\text { Spectrum inefficiency is a significant issue because of the increasingly more } \\
\text { solicitation of transmission capacity by the end clients. Accomplishing high } \\
\text { transmission rates and elevated levels of Quality of Service (QoS) speaks to in any } \\
\text { case an open issue. Long haul Evolution (LTE) has been proposed as the reason for } \\
\text { the fourth era versatile cell systems (4G) that points of the LTE standard are higher } \\
\text { client bit rates, lower delays, expanded range proficiency, diminished expense, and } \\
\text { operational effortlessness. In any case, this innovation is as yet being worked on and } \\
\text { a few open issues must be still explored, for example, obstruction coordination, and } \\
\text { power utilization, assets the board and handover procedures. The point of this work } \\
\text { is to ensure the decrease of intensity utilization utilizing another handover } \\
\text { calculation dependent on green arrangement. Also, the proposed conspire ensures } \\
\text { the minimization of superfluous handovers. In any case, the issue in this technique is } \\
\text { doesn't mulls over of the impact of green strategies in high force hubs. Along these } \\
\text { lines, in the proposed framework the impact of high force hubs are thought of. This } \\
\text { situation is expanded more than one full scale cell and various kinds of low force } \\
\text { hubs, for example, pico-cell and microcells. In this situation, the force sparing is } \\
\text { broke down during the handover strategies. Think about these issues; another } \\
\text { strategy is proposed called Dynamic base Station arranging (DBSP) for } \\
\text { accomplishing vitality productivity. The fundamental idea is to kill a BS individually } \\
\text { that will insignificantly influence the system by utilizing a recently presented thought } \\
\text { of system sway, which considers the extra burden increases brought to its } \\
\text { neighboring BSs. So as to additionally diminish the flagging and execution overhead } \\
\text { over the air and back take, utilize the estimated estimations of system sway as their } \\
\text { choice measurements. A trial result shows that the proposed strategy accomplishes } \\
\text { high vitality proficiency under different situations. In this work the presentation of an } \\
\text { UMTS organize situation is assessed by utilizing different estimations of the priority } \\
\text { bits of the CBR application. } \\
\text { Keywords: Spectrum, UMTS, QoS, hand over }\end{array}$ \\
\hline
\end{tabular}

\section{Introduction}

The new period of correspondence, at present utilized in certain pieces of the world, is Worldwide Interoperability for Microwave Access (WIMAX). It is the most recent innovation which is endorsed by IEEE 802.16 gathering, which is a standard for highlight multi point remote systems administration. Wi-Max vision is to convey "last mile" broadband availability to home or business areas, likewise its information rates are practically identical with Cable and Digital Subscriber line (DSL) rates. It has the capacity which interfaces with the ISP (Internet Service Provider) in any event, when you are meandering outside home or office. The Wi-max innovation is turning into the best approach to deflect the looming emergency of rustic network for example it will be available till the last mile. This paper clarifies about the reason for Wi-max, the investigation of Wi-max frameworks, its suggestions and applications and its remote abilities [1]. 
Envision an innovation that can venture even into the remote regions or suburbia where the entrance to control plants is even troublesome. This advancement innovation will give move paces of various Megabits to the clients inside a scope of a few kilometers. As we probably am aware the interest for remote has raised quickly from 5.7 million of every 2002 to 200 million of every 2006. The worldwide Wimax advertise is developing at the pace of $30 \%$ every year and is required to contact $\$ 2.8$ billion by 2009 from the present $\$ 600$ million. The quantity of Wi-max clients is conjecture ed to each 14.9 million in 2009. Wimax based arrangements are progressively adaptable and made sure about. They offer a remarkable assistance and are a lot of financially savvy. Geographies of urban and rural zones can be constrained by this innovation. Wi-max innovation is multiple times quicker than the third era and multiple times quicker than the remote information rates [2].

WiMAX (Worldwide Interoperability for Microwave Access) measures characterize formal details for arrangement of broadband. Remote metropolitan territory systems as characterized in Wi-MAX gauges give remote broadband access anyplace, whenever, and on basically any gadget. It is an entrance innovation that guarantees high information rates and wide inclusion requiring little to no effort. It permits getting to broadband Internet even while moving at vehicular paces of up to $125 \mathrm{kmph}$. The two primary utilization of Wi-max are Fixed Wi-max, which are point to multi guide empowering broadband access toward homes and workplaces, and Mobile Wi-max, which offers the full portability of cell systems at genuine rates. Additionally the rise of cutting edge remote innovation is going to upgrade the adequacy of the current strategies utilized by open wellbeing. Wi-max has the potential or capacity to furnish India with boundless and incredible Internet get to that can introduce financial development. Better instruction and social insurance, and improved amusement administrations. Wi-max can give Internet access to private clients in rural and "extremely" rustic territories the window to an entirely different world that the now - out of date cabled broadband couldn't offer. Each and every individual who acknowledges opportunity from the shackles of links would adore Wi-max and what more when the new remote association offers advantageous correspondence, modest calls, quick downloads and smooth video spilling [3].

A direct is a division in a transmission medium with the goal that it very well may be utilized to send numerous surges of data. For instance, a radio broadcast may communicate at $91.1 \mathrm{MHz}$ while another radio broadcast may communicate at 91.9 MHz. For this situation, the medium has been partitioned into various frequencies and each radio broadcast is assigned a different recurrence for its communicate. This is known as Frequency Division Multiplexing (FDM). On the other hand, we can dispense each channel a rehashing portion of time to communicate on. This is known as Time Division Multiplexing (TDM). Both TDM and FDM are utilized in computerized correspondence. Access arranges is that segment of the system between the client premise and the system administrator's (typically closest) purpose of quality. It is likewise alluded to as the last mile" or the nearby circle". Web gets to alludes to the methods by which clients associate with the Internet to get to any of the applications that depend on Internet. Basic techniques for Internet access for private use are dial-up, DSL and link modem. Broadband Internet get to, regularly abbreviated to simply broadband, is fast Internet get to [4].

OECD (Organization for Economic Co-activity and Development), in its 2006 OECD Broadband Statistics report, characterizes broadband as download paces of more prominent than or equivalent to $256 \mathrm{kbits} / \mathrm{s}$. US FCC characterizes broadband as download velocities of more noteworthy than $768 \mathrm{kbits} / \mathrm{s}$ [3]. At the point when broadband Internet get to is through the remote medium, it is alluded to as Broadband Wireless Access (BWA). Correspondence made conceivable when the transmitter and receiver(s) are put on skyscraper towers in order to stay away from every single physical hindrance (like trees and structures) between them, is called view (LOS) correspondence. Where LOS correspondence is beyond the realm of imagination (for example at the point when transmitters/beneficiaries are gadgets inside a home), signals transmitted from the collector experience weakening and multi way mutilation (in the wake of skipping of trees and structures). This kind of correspondence is called non view (NLOS) correspondence. At the point when the correspondence is between one sender and one beneficiary, it is called highlight point (PTP) media communications. At the point when the correspondence is between one sender and different beneficiaries, it is called highlight multi point (PMP) broadcast communications. Communicate and multicast are instances of PMP correspondence [5]. 
The 70 Mbps data rate that Wi-MAX provides could be shared among multiple subscribers. Even though IEEE 802.16 standards allow all frequencies between 1 and $66 \mathrm{GHz}$, Wi-MAX standards, to promote compatibility and interoperability between devices manufactured by different vendors, restrict spectrum usage to the frequency ranges mentioned in table 1. Fixed and mobile access types are same as the ones depicted in figure 1. In nomadic/portable access, as against fixed and mobile access types, a subscriber can move from one place to another but his/her Wi-MAX access will be from one location.

\section{Existing techniques}

The framework researches dynamic base station (BS) changing to lessen vitality utilization in remote cell systems. In particular, framework define a general vitality minimization issue relating to BS exchanging that is known to be a troublesome combinatorial issue and requires high computational multifaceted nature just as enormous flagging overhead. The proposed framework presented a for all intents and purposes implementable turning on/off based vitality sparing (SWES) calculation that can be worked in a circulated way with low computational multifaceted nature [6].

In SWES, which BSs get turned on/off individually while guaranteeing clients' QoS. Turning off Algorithm: The choice standard (i.e., organize sway) characterized in (10) just relies upon data for a BS and its neighbouring BSs. In this manner, it is conceivable that the turning off choice can be limited as an issue at every BS. The proposed dispersed turning off calculation is straightforward: the framework data, for example, signal quality and framework load is occasionally shared among BSs and UEs, and every BS decides if it ought to be killed or not. Note that the proposed calculation doesn't require the brought together controller. A key structure rule of the proposed calculation is to kill a BS individually that will insignificantly influence the system by utilizing a recently presented idea of system sway, which considers the extra burden increases brought to its neighbouring BSs. So as to additionally lessen the flagging and usage overhead over the air and backhaul, the framework proposes three other heuristic variants of SWES that utilization the surmised estimations of system sway as their choice measurements [7].

The framework considers vitality effectiveness in cell organize arranging. Framework present another boundary for traffic estimation, which is low traffic time proportion. So as to turn off more cells for deficient cell zooming, two arrangements are doable: to send littler yet more cells or to execute inclusion expansion innovations. cells couldn't zoom to unendingness because of limitation of transmit power. After customary arranging, we would get list of last doable plan $\mathrm{K}_{-}$max We could choose whether it is vitality productive to convey littler however more cells to empower kth $\left(\mathrm{k}>\mathrm{K} \_\right.$max $)$turning off plan, say $(\mathrm{m} ; \mathrm{n})$ - off plan. Proposed framework centre around previous answer for decide cell designs and propose an assessment strategy to decide if certain cell organization is vitality productive and how much vitality it could spare, contrasted and conventional arranging. It is demonstrated that when cell zooming proportion is arriving at adequate for certain turning off plan, conveying more cells could be more vitality effective. Altering cell sizes to diminish vitality utilization impact of cell size on the vitality expended organize expecting base station innovations of today and what's to come. Expand cell size too little or too enormous can fundamentally build the force utilization. Multilayer design smaller cells are progressively productive for supporting higher limit yet because of the fixed force utilization, they become less vitality effective when the interest is low.

In Energy proficiency of a two layer cell arranges the "Fixed" plot chooses a fixed cell range which limits all out force utilization while fulfilling limit imperatives. The "Ideal" plot shows the perfect situation, where the cell sweep changes to be the ideal as far as vitality at consistently. This plan spares the most force (see Table 2) and is an upper-destined for any cell breathing plan we utilize. At long last, the more viable "Dynamic" conspire shows the force utilization of our proposed two-layer engineering. This plan picks two radii that limit all out force utilization throughout the day and switches between these two radii relying upon the traffic at each hour. The framework show that the ideal cell size from a vitality point of view relies upon various components, including base station innovation, information rates, and traffic requests.

Vitality Efficiency in cell portable radio systems has as of late increased incredible enthusiasm for the examination network. Other than the constructive outcome on worldwide environmental change, bringing down 
force utilization of versatile systems is advantageous regarding diminishing the operational expense for arrange administrators. In such manner, the improvement of more vitality productive equipment and programming segments aside, impact of various sending systems on vitality proficiency are likewise concentrated in the writing. The proposed framework explores the vitality productivity enhancements through various heterogeneous systems for both uniform and non-uniform traffic circulation situations [8].

The framework considers a hexagonal network of 19 locales with a focal reference cell encompassed by two levels of interferers. In this design, full scale base stations are sent in the cell, transmitting through Omni directional reception apparatuses, while at least one little low force base stations; either miniaturized scale, Pico and WLAN are conveyed at the phone edge with fixed cell run and transmit power. Framework additionally think about thick large scale situation, where the sent base stations at cell edge are full scale base stations with fixed force. It is accepted that these base stations don't add to large scale cell inclusion [9].

WiMAX is fourth era remote correspondence innovation WiMAX is cheap foundation more extensive inclusion and high information rate make WiMAX overall interoperability for microwave access to be one of the most creating innovation for remote last mile association. Improve versatility remote access is planned, for example, 802.16e to work progressing with no meddle of administrations. Versatile WiMAX support meandering administrations in metropolitan and local system, so permit portable availability to portable client [10].

\section{Proposed Methodology}

Versatile executives understood the handover work among the neighbouring BS. Handover alludes to the activity of changing over the remote connection interfacing MS to one BS to another BS. In versatile Wi-MAX, two sorts of handover, first is hard handover and second iis delicate handover. In the hard handover discharges the past connection before making another call to another base station. In the versatile Wi-MAX just help a hard handover, though 802.16e help a the two sorts of handover is hard and delicate handover.

Versatile WiMAX is favorable in supporting low-inactivity information, video and ongoing voice administrations for portable client at rapid .In this paper the Performance investigation of versatile WiMAX handover with different speed utilizing CBR application has been embraced. In the proposed framework, Dynamic base Station arranging (DBSP) is introduced for improving vitality effectiveness during the handover procedure between the large scale cell and various kinds of low force hubs, for example, pico cell and microcells. Since the current strategy doesn't thinks about of the impact of green strategies in high force hubs.

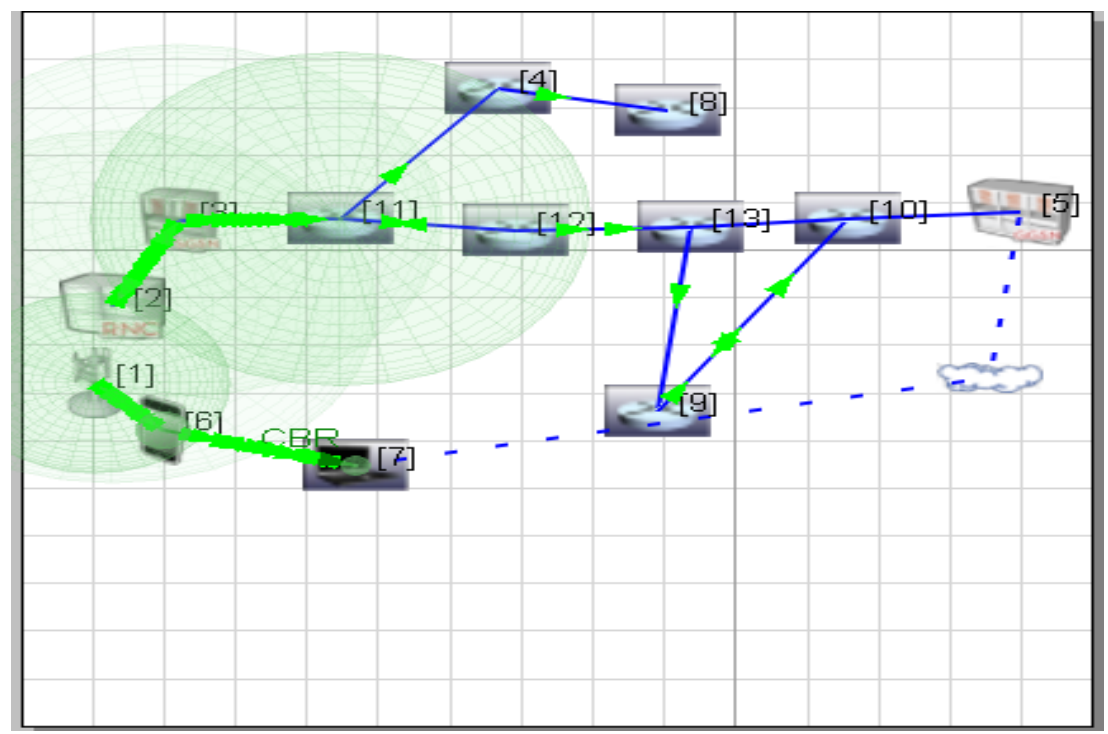

Fig 1: Basic UMTS Structure with handover technique 
The proposed framework considers the handover procedure between the large scale cell and various sorts of low force hubs, for example, pico cell and microcells. The primary idea is to kill a BS individually that will insignificantly influence the system by utilizing a recently presented thought of system sway, which considers the extra burden increases brought to its neighboring BSs. The general issue of vitality sparing with BS exchanging is figured as a combinatorial issue. To ideally take care of this intricate issue, a focal controller is required. In this work, present an idea of the system sway for a particular BS, which is characterized as how much the turning off of this BS will influence the system. The system sway is made out of deterministic boundaries at every BS, for example, inward (own) and outer (neighboring BS's) framework burden, and generally framework boundaries, for example, the traffic profile. Notwithstanding that, the exchanging impact BSs is likewise thought of. In view of the exercise gained from this straightforward case, consider which BSs get turned on/off individually while guaranteeing clients' QoS.

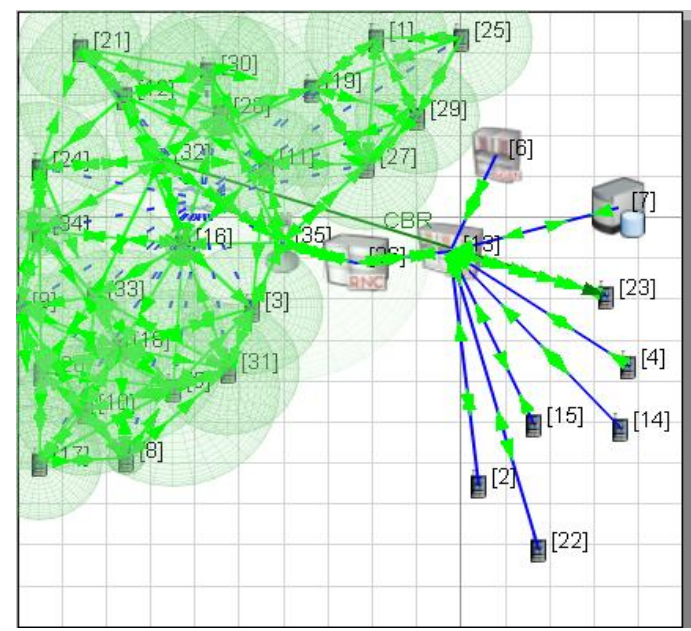

Fig 2: UMTS Structure of the proposed system

\section{Simulation Results}

In order to oversee QoS assets, the SAE engineering coordinates an instructive QoS Information Function (QIF) work that connects with all individual system models included inside the IMS stage. This QoS asset the board arrangement is an outside piece of the system, in light of the utilization and communication between a focal element and periferic components. Despite the fact that in the fresh start approach the asset the board and the QoS support are viewed as an incorporated piece of interchanges arranges, this reality isn't reflected in the attributes of current frameworks or by the UMTS organize engineering. Hence, the point of view of an autonomic asset the board in an UMTS arrange proposed in this paper recommends the need of including extra data at the degree of the focal UMTS systems components utilizing virtualization method. Reproductions are performed utilizing NS-2 Simulator.

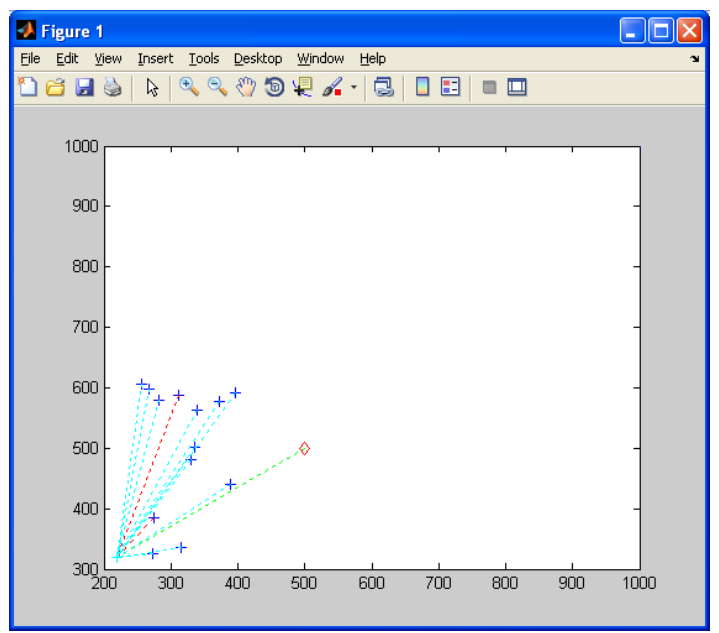

Fig 3: Performance of the proposed algorithm 
System virtualization speaks to an elevated level reflection process that covers the execution and physical system arrangement subtleties. Permitting concurrence of various virtual designs overlaid on a typical substrate truly shared, organize virtualization guarantees adaptability and security, advancing assorted variety and expanded administration limit. Along these lines, UMTS center system hubs act independently, having the option to detect the earth, to see the changes, to comprehend inward changes and to respond in a shrewd way by choosing the ideal way as per application prerequisites. To exhibit this, local UMTS QoS support is broke down in contrast with the capability of the autonomic administration offered through system virtualization, utilizing QualNet arrange test system. Subsequently, on account of an autonomic administration framework, the proposed investigation situations feature the capacity of choosing the best course as per the source application limitations regarding most extreme satisfactory start to finish delay.

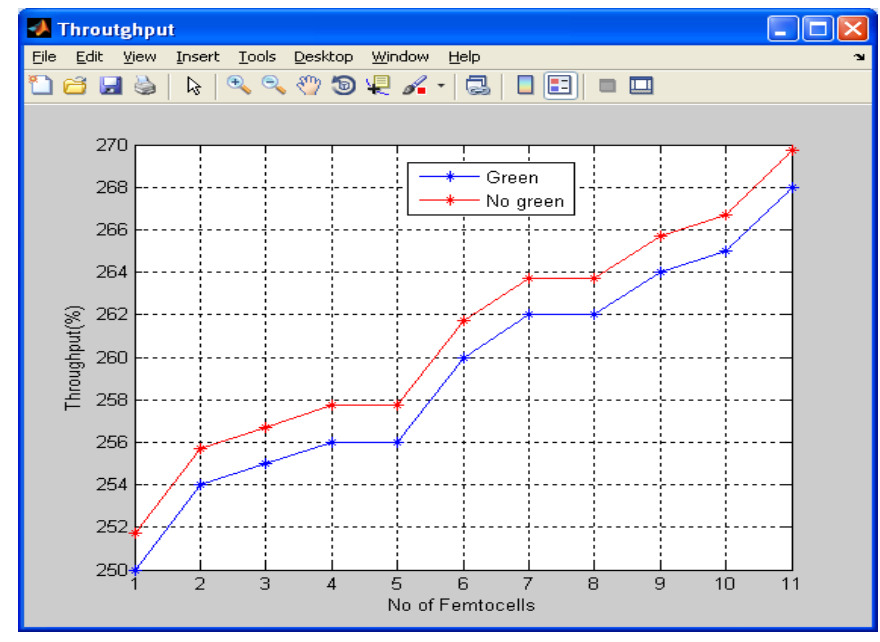

Fig 4: Simulation result showing throughput of handover algorithm

Considering a reasonable UMTS RAN space displaying, the paper intends to explore the potential advantages that could live from the mix of the autonomic asset the executives dependent on the virtualization procedure in current UMTS IP PS arrange area design. In view of a goal QoS/QoE execution examination, the maximum furthest reaches of local UMTS QoS support was broke down, in a first stage, regarding normal start to finish delay, normal jitter and throughput. The capacity of the UMTS traffic classes to offer quality help for steady piece rate time basic applications was contrasted and the QoS support came about because of the use of autonomic asset the board. The got outcomes recommend that, since it is nearer to the application needs and thinking about the system setting, such an autonomic asset the board could improve the local UMTS QoS support. Thus, the improved QoS/QoE backing would conquer the circumstances wherein the existent QoS component would not acknowledge the administration itself.

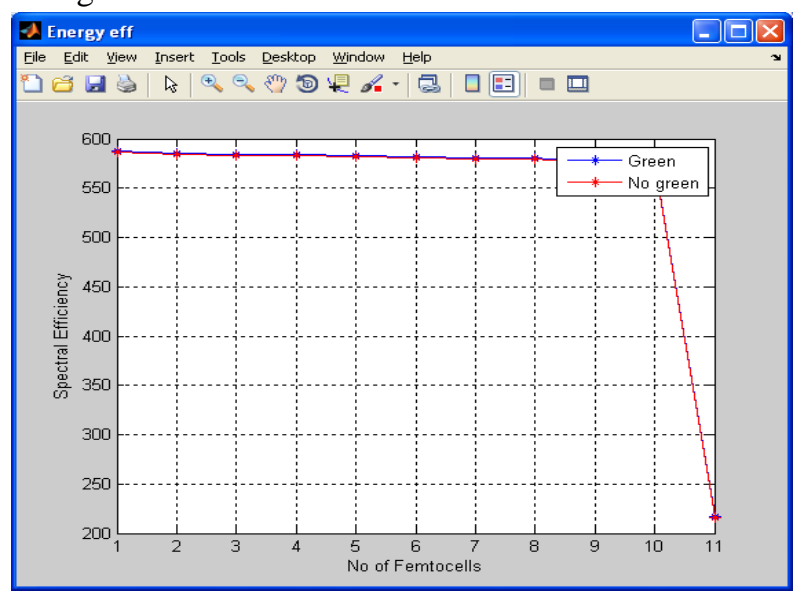

Fig 5: Simulation result showing energy efficiency of handover algorithm 


\section{Conclusion}

This exploration article proposes a handover calculation dependent on green strategies for UMTS designs so as to productively oversee handover system ensuring power sparing. Likewise, the calculation permits the decrease of pointless and regular handovers. The proposed Dynamic base Station booking calculation presented for increment vitality productivity by both turning BSs on/off and adaptively modifying their transmission power as per the current traffic conditions. A trial result shows that the proposed technique accomplishes high vitality effectiveness under different situations. A potential answer for improve the handover execution in low force hubs. A handover Dynamic base Station arranging (DBSP) is introduced for improving vitality effectiveness during the handover procedure between the large scale cell and various kinds of low force hubs, for example, picocell and microcell. Since the current technique doesn't contemplates of the impact of green approaches in high force hubs. As a piece of the further work, the creators expect to expand this arrangement likewise for the radio access some portion of the cell systems in various parametric conditions and furthermore the quantity of hubs can be fluctuated with their particular good ways from the server.

\section{References}

[1] Tianyi Qin, Drivers drowsiness detection in embedded system, IEEE International Conference on Vehicular Electronics and Safety, 2007. ICVES

[2] Ian F. Akyildiz, David M. Gutierrez-Estevez, and Elias Chavarria Reyes. 2010. The evolution to 4G cellular systems: LTE-Advanced. Phys. Commun. 3, 4 (December 2010), 217-244.

[3] G. Araniti, V. Scordamaglia, M. Condoluci, A. Molinaro, A. Iera, "Efficient Frequency Domain Packet scheduler for Point-to-Multipoint transmissions in LTE networks," Communications (ICC), 2012 IEEE International Conference on , vol., no., pp.4405,4409, 10-15 June 2012.

[4] M. Condoluci, G. Araniti, A. Molinaro, A. Iera, J. Cosmas, "On the impact of frequency selectivity on multicast subgroup formation in 4G networks," Broadband Multimedia Systems and Broadcasting (BMSB), 2013 IEEE International Symposium on , vol., no., pp.1,6, 5-7 June 2013.

[5] A. Khandekar, N. Bhushan, Ji Tingfang, V. Vanghi, "LTE-Advanced: Heterogeneous networks," Wireless Conference (EW), 2010 European , vol., no., pp.978,982, 12-15 April 2010.

[6] Yan Chen, Shunqing Zhang, Shugong Xu, G.Y. Li, "Fundamental tradeoffs on green wireless networks," Communications Magazine, IEEE, vol.49, no.6, pp.30,37, June 2011.

[7] S. Frattasi, R.L. Olsen, M. De Sanctis, F. Fitzek, R. Prasad, "Heterogeneous services and architectures for next-generation wireless networks," Wireless Communication Systems, 2005. 2nd International Symposium on , vol., no., pp.213,217, 5-7 Sept. 2005.

[8] I. Bisio, M. Marchese, "Power Saving Bandwidth Allocation over GEO Satellite Networks," Communications Letters, IEEE, vol.16, no.5, pp.596,599, May 2012.

[9] J. Anand, A. S. Buttar and R. Kaur, "Handover Triggering Based Spectrum Handover Approach in Cognitive Radio Network: A Survey," 2018 Second International Conference on Electronics, Communication and Aerospace Technology (ICECA), Coimbatore, 2018, pp. 830-835, DOI: 10.1109/ICECA.2018.8474549.

[10] N. K. Panigrahy and S. C. Ghosh, "Analysing the Effect of Soft Handover on Handover Performance Evaluation Metrics Under Load Condition," in IEEE Transactions on Vehicular Technology, vol. 67, no. 4, pp. 3612-3624, April 2018, DOI: 10.1109/TVT.2017.2786342. 